PROCEEDINGS OF THE

AMERICAN MATHEMATICAL SOCIETY

Volume 128, Number 6, Pages 1733-1742

S 0002-9939(99)05536-7

Article electronically published on November 23, 1999

\title{
LONG TIME HEAT DIFFUSION ON HOMOGENEOUS TREES
}

\author{
GUIA MEDOLLA AND ALBERTO G. SETTI \\ (Communicated by Christopher D. Sogge)
}

\begin{abstract}
Let $\mathfrak{X}$ be a homogeneous tree of degree $q+1, q \geq 2, \mathcal{L}$ the Laplace operator of $\mathfrak{X}$ and $h_{t}(x)$ the fundamental solution of the heat equation $\left(\partial_{t}+\mathcal{L}\right) u=0$ on $\mathfrak{X}$. We show that the heat kernel $h_{t}(x)$ is asymptotically concentrated in an annulus moving to infinity with finite speed $R_{1}=(q-1) /(q+1)$. Asymptotic concentration of heat in the $L^{p}$ norm is also investigated.
\end{abstract}

\section{INTRODUCTION}

Let $\mathfrak{X}$ be a homogeneous tree of degree $q+1$, that is, a connected graph with no loops in which every vertex is adjacent to $q+1$ other vertices; in particular if $q$ is equal to one, then the tree may be identified with the integers $\mathbb{Z}$.

We will write $x \sim y$ if $x$ and $y$ are adjacent. The tree is endowed with a natural distance function $d$, where $d(x, y)$ is the number of edges in the unique path from $x$ to $y$, and by a natural Laplace operator $\mathcal{L}$ defined by

$$
\mathcal{L} f(x)=\frac{1}{q+1} \sum_{y \sim x}[f(x)-f(y)] .
$$

If we denote by $L^{p}(\mathfrak{X})$ the Lebesgue spaces on $\mathfrak{X}$ with respect to counting measure, then $\mathcal{L}$ is easily seen to be bounded on $L^{p}(\mathfrak{X})$ for every $1 \leq p \leq+\infty$, and selfadjoint on $L^{2}(\mathfrak{X})$, and therefore the heat operator $\mathcal{H}_{t}$, which is spectrally defined on $L^{2}(\mathfrak{X})$ by

$$
\mathcal{H}_{t} f=\int_{\sigma_{2}(\mathcal{L})} e^{-t \lambda} d P_{\lambda} f \quad \forall t \in(0,+\infty) \quad \forall f \in L^{2}(\mathfrak{X}),
$$

where $\sigma_{2}(\mathcal{L})$ denotes the $L^{2}$ spectrum of $\mathcal{L}$, and $P_{\lambda}$ its spectral resolution, is also given by the series

$$
\mathcal{H}_{t}=\sum_{k=0}^{+\infty} \frac{(-t)^{k} \mathcal{L}^{k}}{k !}
$$

and the series is absolutely convergent in the $L^{p}(\mathfrak{X})$ operator norm.

Received by the editors July 14, 1998.

2000 Mathematics Subject Classification. Primary 43A85, 35K05; Secondary 39A12.

Key words and phrases. Homogeneous trees, heat equation, long time heat diffusion.

The first author acknowledges financial support through a post-doctoral fellowship at the Politecnico di Milano. 
If $f$ is in $L^{p}(\mathfrak{X})$, then $u(x, t)=\mathcal{H}_{t} f(x)$ is the norm analytic solution in $L^{p}$ of the Cauchy problem for the heat equation for $\mathcal{L}$

$$
\left\{\begin{array}{l}
\left(\partial_{t}+\mathcal{L}\right) u=0 \quad \\
u(x, 0)=f(x)
\end{array} \quad \forall(x, t) \in \mathfrak{X} \times \mathbb{R}^{+},\right.
$$

which is the discrete analogue of the classical heat equation on Euclidean space and more generally on Riemannian manifolds.

Denoting by $\delta_{y}$ the Dirac measure at the point $y$ of $\mathfrak{X}$, the heat kernel is defined by $h_{t}(x, y)=\left(\mathcal{H}_{t} \delta_{y}\right)(x)$ so that, given an arbitrary function $f$ in $L^{p}(\mathfrak{X})$, we have

$$
\mathcal{H}_{t} f(x)=\mathcal{H}_{t}\left(\sum_{y} f(y) \delta_{y}\right)(x)=\sum_{y} f(y) h_{t}(x, y) .
$$

The heat kernel $h_{t}(x, y)$ is the solution of the heat equation with initial condition $\delta_{y}$ and describes the diffusion of a unit amount of heat originally concentrated at the point $y$.

Note that the self adjointness of $\mathcal{H}_{t}$ implies that $h_{t}(x, y)$ is symmetric in $x$ and $y$, and then the identity $\mathcal{H}_{t} 1=1$ yields

$$
1=\left(\mathcal{H}_{t} 1\right)(y)=\sum_{x} h_{t}(y, x)=\sum_{x} h_{t}(x, y) \quad \forall t \geq 0, y \in \mathfrak{X},
$$

showing that heat is conserved.

It is also not hard to see that $h_{t}(x, y)$ depends only upon the distance between $x$ and $y$. Moreover it is strictly positive for all $x, y$ in $\mathfrak{X}$ (see, e.g., the lower estimate in Theorem 2 below). The last property expresses the fact that heat propagates instantaneously on $\mathfrak{X}$, which is a well known feature of heat diffusion in the continuous setting.

It was observed by E. B. Davies ([D], Corollary 5.3.7) that the heat kernel $h_{t}(x, y)$ on $n$ dimensional real hyperbolic space satisfies

$$
\int_{R t \leq d(x, y) \leq R^{\prime} t} h_{t}(x, y) d x, \quad \text { as } t \rightarrow \infty,
$$

whenever $R<n-1<R^{\prime}$. This means that, even though heat propagates instantaneously, heat produced at a point $y$ does not propagates homogeneously, but is asymptotically concentrated in an annulus that moves to infinity with finite speed.

In AS this property of heat diffusion was extended to Riemannian symmetric spaces of the noncompact type, and also to a larger class of Riemannian manifolds with exponential volume growth, and bottom of the $L^{2}$ spectrum of the Laplacian bounded away from 0 . In the symmetric space setting, a probabilistic interpretation of this phenomenon was subsequently given by M. Babillot $[\mathrm{B}]$.

The purpose of this note is to show that a similar property holds for the heat diffusion on $\mathfrak{X}$. Indeed we prove the following

Theorem 1. Assume that $q$ is greater than or equal to two. Let $R_{1}=(q-1) /(q+1)$ and let $r(t)$ be a positive function such that

$$
\frac{r(t)}{t^{1 / 2}} \rightarrow \infty \quad \text { as } t \rightarrow \infty
$$


Then for every $y$ in $\mathfrak{X}$

$$
\sum_{\substack{x \\ R_{1} t-r(t) \leq d(x, y) \leq R_{1} t+r(t)}} h_{t}(x, y) \rightarrow 1 \quad \text { as } t \rightarrow \infty .
$$

The proof of (1) depends on some precise estimates for the heat kernel on $\mathfrak{X}$ obtained in [CMS] (see also $[\overline{\mathrm{P}}$ ). This is the same approach that was used in [AS] to prove the symmetric space result. It may be interesting to note that in $\overline{\mathrm{AS}}$, the extension of the result to Riemannian manifolds used wave equation techniques, and was based in particular on the finite propagation speed of the (modified) wave equation. These techniques fail in our setting, essentially because solutions of the wave equation on $\mathfrak{X}$ do not have finite speed of propagation [MS]. A more direct approach seems therefore unavoidable.

This gives further evidence to the fact, already apparent in [CMS], that there is a very close analogy between heat diffusion on homogeneous trees and heat diffusion on hyperbolic space. By contrast, the results of [MS] indicate that the features of wave propagation in the continuous and discrete settings are quite different.

It should also be noted that the validity of (1) is strictly connected to the exponential volume growth of the tree. Indeed if $q=1$, so that we are considering the heat equation on $\mathbb{Z}$, the following result holds.

Theorem 2. Assume that $q=1$, and let $r_{i}(t), i=1,2$ be two positive functions satisfying $r_{1}(t) / \sqrt{t} \rightarrow 0$ and $r_{2}(t) / \sqrt{t} \rightarrow+\infty$. Then for every $y$ in $\mathbb{Z}$

$$
\sum_{r_{1}(t) \leq d(x, y) \leq r_{2}(t)} h_{t}(x, y) \rightarrow 1 \quad \text { as } t \rightarrow \infty .
$$

In particular, for every pair of constants $R<R^{\prime}$ and every positive $\alpha \neq 1 / 2$,

$$
\sum_{\substack{x \\ R t^{\alpha} \leq d(x, y) \leq R^{\prime} t^{\alpha}}} h_{t}(x, y) \rightarrow 0 \quad \text { as } t \rightarrow \infty,
$$

showing that no analogue of (1) may hold. This corresponds exactly to the result valid for the heat kernel on Euclidean space.

Finally, we remark that the same techniques that lead to Theorem 1 may be used to show that a phenomenon of asymptotic heat concentration also occurs with respect to $L^{p}$ norm for $1<p \leq+\infty$. Theorem 1 is the $p=1$ version of this more general result. Referring to Remark 1 below for a precise formulation, here we only stress that even for $p>1$ the features of the $L^{p}$ heat propagation match exactly those obtained in $[\mathrm{AJ}]$ in the symmetric space setting.

\section{NotATION AND PRELIMINARY RESULTS}

For ease of notation we are going to use the variable constant convention, and denote by $C$ constants that may change from place to place, and depend only on factors quantified before their occurrence.

Given two functions $f(t)$ and $g(t)$, both defined on a set $\mathbf{D}$, we say that $f(t) \sim$ $g(t)$ for all $t$ in $\mathbf{D}$ if there exist positive constants $C$ and $C^{\prime}$ such that

$$
C f(t) \leq g(t) \leq C^{\prime} f(t) \quad \forall t \in \mathbf{D} .
$$

The expression $f(t) \asymp g(t)$ as $t$ tends to $t_{0}$ means that

$$
g(t)=f(t)(1+o(1)) \quad \text { when } t \rightarrow t_{0} .
$$


Let $o$ be a fixed reference point on $\mathfrak{X}$. We say that a function $f$ on $\mathfrak{X}$ is radial if $f(x)$ depends only on the distance $d(o, x)$, also denoted by $|x|$. Clearly, if $q=1$, one may choose $o$ as the vertex 0 , and then $|x|$ is exactly the absolute value of $x$.

We explicitly note that for $q \geq 2$, the cardinality of the set $\{x:|x|=n\}$ is equal to 1 if $n=0$ and to $(q+1) q^{n-1}$, for every $n \geq 1$.

For every $x$ in $\mathfrak{X}$ let $h_{t}(x)$ be defined by $h_{t}(x)=h_{t}(x, o)$. In the sequel we will refer to this function as the heat kernel of the tree, and, to avoid ambiguity, we will denote by $h_{t}^{\mathbb{Z}}$ the heat kernel on $\mathbb{Z}$. Since $h_{t}(x, y)$ depends only upon $d(x, y)$, if follows that $h_{t}(x)$ is radial (and $h_{t}^{\mathbb{Z}}$ is even), and that (1) holds if and only if

$$
\sum_{R_{1} t-r(t) \leq|x| \leq R_{1} t+r(t)} h_{t}(x) \rightarrow 1 \quad \text { as } t \rightarrow+\infty .
$$

An entirely analogous statement of course holds in the case of (2).

A detailed study of the heat operator and of the heat kernel was carried out in [CMS which we refer to for a complete discussion of notation and results.

We only recall the following two key lemmas. The first lemma gives uniform pointwise estimates for $h_{t}^{\mathbb{Z}}$, and depends on a formula expressing $h_{t}^{\mathbb{Z}}$ in terms of modified Bessel functions, and from the uniform asymptotic expansion of modified Bessel functions of large order.

Lemma 3 (CMS], Theorem 2.3). Let $\xi: \mathbb{R}^{+} \rightarrow \mathbb{R}$ and $F: \mathbb{Z} \times \mathbb{R}^{+} \rightarrow \mathbb{R}$ denote the functions defined by the rules

$$
\xi(z)=\left(1+z^{2}\right)^{1 / 2}+\log \left(\frac{z}{1+\left(1+z^{2}\right)^{1 / 2}}\right)
$$

and

$$
F(j, t)= \begin{cases}(2 \pi)^{-1 / 2} \frac{\exp [-t+|j| \xi(t /|j|)]}{\left(1+j^{2}+t^{2}\right)^{1 / 4}}, & j \neq 0, \\ (2 \pi)^{-1 / 2}\left(1+t^{2}\right)^{-1 / 4}, & j=0 .\end{cases}
$$

Then $h_{t}^{\mathbb{Z}}(j) \asymp F(j, t)$ as $j^{2}+t^{2}$ tends to $\infty$, and $h_{t}^{\mathbb{Z}}(j) \sim F(j, t)$ for all $j$ in $\mathbb{Z}$ and $t$ in $\mathbb{R}^{+}$.

The second lemma follows from a formula (CMS, Proposition 2.5 (ii)) relating the heat kernel $h_{t}(x)$ to the heat kernel on $\mathbb{Z}$ which is analogous to the relationship between the heat kernel of a symmetric space and that of a maximal flat submanifold.

Lemma 4 ([CMS], Proposition 2.5 (iii)). For all $t$ in $\mathbb{R}^{+}$and $x$ in $\mathfrak{X}$ the following holds:

$$
h_{t}(x) \sim \frac{e^{-b_{2} t}}{t}(1+|x|) q^{-|x| / 2} h_{t \gamma(0)}^{\mathbb{Z}}(|x|+1)
$$

where $\gamma$ is the entire function defined by the formula

$$
\gamma(z)=\frac{q^{1 / 2}}{q+1}\left(q^{i z}+q^{-i z}\right)
$$

and $b_{2}=1-\gamma(0)=\inf \left(\sigma_{2}(\mathcal{L})\right)$

Clearly, Lemmas 3 and 4 yield uniform upper and lower estimates for $h_{t}(x)$. 


\section{Proof of the theorems}

Proof of Theorem 1. Observe that without loss of generality we may suppose that $r(t)<R_{1} t$. Since $h_{t}$ has total sum 1, it is equivalent to prove that

$$
\sum_{|x| \leq R_{1} t-r(t)} h_{t}(x) \rightarrow 0 \quad \text { as } t \rightarrow \infty
$$

and

$$
\sum_{|x| \geq R_{1} t+r(t)} h_{t}(x) \rightarrow 0 \quad \text { as } t \rightarrow \infty .
$$

To prove (i) we must show that

$$
\sum_{n \leq R_{1} t-r(t)} \sum_{|x|=n} h_{t}(x) \rightarrow 0 \quad \text { as } t \rightarrow \infty .
$$

According to Lemma 4 we have

$$
\sum_{|x|=n} h_{t}(x) \sim \frac{e^{-(1-\gamma(0)) t}}{t}(1+n) q^{n / 2} h_{t \gamma(0)}^{\mathbb{Z}}(n+1),
$$

whence, setting $n+1=d$, the sum in (i) becomes

$$
\sum_{0<d \leq R_{1} t-r(t)+1} \frac{e^{-(1-\gamma(0)) t}}{t} m q^{(m-1) / 2} h_{t \gamma(0)}^{\mathbb{Z}}(m) .
$$

Since $t^{-1 / 2}(r(t)-1) \rightarrow \infty$, with only slight abuse of notation we may replace $r(t)-1$ with $r(t)$.

Now, recalling that $h_{t}^{\mathbb{Z}}(j) \sim F(j, t)$ and substituting the definition of the function $F$, we deduce that we need to estimate the series

$$
\sum_{d \leq R_{1} t-r(t)} \frac{d}{t\left[d^{2}+\gamma^{2}(0) t^{2}\right]^{1 / 4}} \exp \{g(d, t)\}
$$

as $t \rightarrow \infty$, where

$$
g(s, t)=-t+\frac{s}{2} \log q+\left(s^{2}+\gamma^{2}(0) t^{2}\right)^{1 / 2}+s \log \left(\frac{\gamma(0) t}{s+\left[s^{2}+\gamma^{2}(0) t^{2}\right]^{1 / 2}}\right) .
$$

Differentiating with respect to $s$ yields

$$
g^{\prime}(s, t)=\frac{1}{2} \log q+\log [\gamma(0) t]-\log \left[s+\left(s^{2}+\gamma^{2}(0) t^{2}\right)^{1 / 2}\right]
$$

and

$$
g^{\prime \prime}(s, t)=-\frac{1}{\sqrt{s^{2}+\gamma^{2}(0) t^{2}}}
$$

from which it follows that the function $s \mapsto g(s, t)$ attains its absolute maximum at the point $R_{1} t=\frac{q-1}{q+1} t$. Expanding $g(s, t)$ around $R_{1} t$ we write

$$
g(d, t)-g\left(R_{1} t, t\right)=\left(d-R_{1} t\right) g^{\prime}\left(R_{1} t, t\right)+\int_{R_{1} t}^{d}(d-s) g^{\prime \prime}(s, t) d s
$$


and since $g\left(R_{1} t, t\right)=g^{\prime}\left(R_{1} t, t\right)=0$ and $g^{\prime \prime}(s, t) \leq-1 / t$ in $\left[0, R_{1} t\right]$, we have

$$
\begin{aligned}
g(d, t) & =\int_{d}^{R_{1} t}(s-d) g^{\prime \prime}(s, t) d s \\
& \leq-\frac{1}{2 t}\left(R_{1} t-d\right)^{2} \leq-\frac{1}{2 t}\left(d_{1}-d\right)^{2}-\frac{1}{2 t} r^{2}(t)
\end{aligned}
$$

where we have set, for ease of notation, $d_{1}=d_{1}(t)=R_{1} t-r(t)$. In the range of $d$ under consideration we also have

$$
\frac{d}{\left[d^{2}+\gamma^{2}(0) t^{2}\right]^{1 / 4}} \leq C \frac{1}{t^{1 / 2}}
$$

and we conclude that the sum in (4) is bounded above by

$$
\frac{C}{t^{1 / 2}} e^{-\frac{1}{2 t} r^{2}(t)} \sum_{d \leq d_{1}} e^{-\frac{1}{2 t}\left(d_{1}-d\right)^{2}}
$$

Since the function $s \mapsto e^{-\frac{1}{2 t}\left(d_{1}-s\right)^{2}}$ is increasing in $\left[0, d_{1}\right]$, the latter sum is in turn estimated by

$$
\frac{C}{t^{1 / 2}} e^{-\frac{1}{2 t} r^{2}(t)}\left(\int_{0}^{d_{1}} e^{-\frac{1}{2 t}\left(d_{1}-s\right)^{2}} d s+1\right) .
$$

Performing the change of variables $u=\frac{d_{1}-d}{\sqrt{2 t}}$ the integral above becomes

$$
\sqrt{2 t} \int_{0}^{d_{1} / \sqrt{2 t}} e^{-u^{2}} d u \leq \sqrt{2 t} \int_{0}^{+\infty} e^{-u^{2}} d u \leq C \sqrt{t}
$$

and (i) follows.

We now prove (ii). Arguing as in the proof of (i) shows that we need to prove that

$$
\sum_{d \geq R_{1} t+r(t)} \frac{d}{t\left[d^{2}+\gamma^{2}(0) t^{2}\right]^{1 / 4}} e^{g(d, t)} \rightarrow 0 \quad \text { as } t \rightarrow+\infty .
$$

Let $d_{2}=R_{1} t+r(t)$. If $d \geq d_{2}$, it follows from (6) that

$$
\begin{aligned}
g(d, t)=\int_{R_{1} t}^{d}(d-s) g^{\prime \prime}(s) d s & =\int_{R_{1} t}^{d_{2}}(d-s) g^{\prime \prime}(s) d s+\int_{d_{2}}^{d}(d-s) g^{\prime \prime}(s) d s \\
& =I_{1}(d, t)+I_{2}(d, t) .
\end{aligned}
$$

We begin estimating $I_{1}(d, t)$. Since $d_{2} \leq 2 R_{1} t$, in this case $R_{1} t \leq s \leq 2 R_{1} t$ and, by $(5), g^{\prime \prime}(s) \leq-C / t$. Since $d-s \geq d_{2}+r(t)-s$, we obtain

$$
\int_{R_{1} t}^{d_{2}}(d-s) g^{\prime \prime}(s) d s \leq-\frac{C}{t} \int_{R_{1} t}^{d_{2}}\left(d_{2}-s\right) d s=-\frac{C}{2 t} r^{2}(t) .
$$

Now let us consider $I_{2}(d, t)$. In the range $m_{2}<s<d$ we have $g^{\prime \prime}(s) \leq-\tilde{C} / s$ so that

$$
\begin{aligned}
\int_{d_{2}}^{d}(d-s) g^{\prime \prime}(s) & \leq-\tilde{C} \int_{d_{2}}^{d} \frac{d-s}{s} d s \\
& =-\tilde{C}\left(d \log \frac{d}{d_{2}}-\left(d-d_{2}\right)\right)
\end{aligned}
$$


and therefore

$$
g(d, t) \leq-C\left(\frac{r^{2}(t)}{t}+d \log \frac{d}{d_{2}}-\left(d-d_{2}\right)\right) .
$$

Since it is clear that for $d \geq R_{1} t$

$$
\frac{d}{t} \frac{1}{\left[d^{2}+\gamma^{2}(0) t^{2}\right]^{1 / 4}} \leq \frac{\sqrt{d}}{t}
$$

we deduce that

$$
\begin{aligned}
\sum_{d \geq R_{1} t+r(t)} \frac{d}{t\left[d^{2}+\gamma^{2}(0) t^{2}\right]^{1 / 4}} & e^{g(d, t)} \\
& \leq \frac{e^{-C r^{2}(t) / t}}{t} \sum_{d \geq d_{2}} \sqrt{d} e^{-C\left[d \log \frac{m}{d_{2}}-\left(d-d_{2}\right)\right]} .
\end{aligned}
$$

Setting $u=d / d_{2}$, it follows that

$$
\begin{aligned}
\sqrt{d} e^{-C\left[d \log \frac{d}{d_{2}}-\left(d-d_{2}\right)\right]} & =\sqrt{d_{2}} \sqrt{u} e^{-C d_{2}[u \log u-(u-1)]} \\
& \leq \sqrt{d_{2}} e^{-C d_{2}[u \log u-(u-1)] / 2}
\end{aligned}
$$

showing that the right-hand side of (7) is bounded above by

$$
\frac{\sqrt{d_{2}}}{t} e^{-C r^{2}(t) / t} \sum_{d \geq d_{2}} e^{-C d_{2}\left[\frac{d}{d_{2}} \log \frac{d}{d_{2}}-\left(\frac{d}{d_{2}}-1\right)\right] / 2} .
$$

Again we estimate the series above with the corresponding integral, and change variables to get

$$
\begin{array}{r}
\sum_{d \geq d_{2}} e^{-C d_{2}\left[\frac{d}{d_{2}} \log \frac{d}{d_{2}}-\left(\frac{d}{d_{2}}-1\right)\right] / 2} \leq \int_{d_{2}}^{\infty} e^{-C d_{2}\left[\frac{s}{d_{2}} \log \frac{s}{d_{2}}-\left(\frac{s}{d_{2}}-1\right)\right] / 2} d s+1 \\
=d_{2} \int_{1}^{\infty} e^{-C d_{2}[u \log u-u+1] / 2} d u+1 \leq \bar{C} \sqrt{d_{2}}+1
\end{array}
$$

where the last inequality follows from an application of Laplace method (see, e.g., [E], pp. 36-37).

Substituting this in (7), and recalling that $d_{2} \leq 2 R_{1} t$, we conclude that the right-hand side of (7) is bounded above by

$$
C^{\prime} e^{-C r^{2}(t) / t}
$$

which tends to zero as $t \rightarrow \infty$, as required to finish the proof.

Remark 1. Similar techniques may be used to show that heat asymptotically concentrates with respect to $L^{p}$ norm also for $1<p \leq+t y$. In this case the $L^{p}$ norm of $h_{t}$ is not independent of $t$. Indeed, for every $1 \leq p \leq+\infty$, denote by $\delta(p)$ and $p^{\prime}$ the number $1 / p-1 / 2$ and the conjugate index $p /(p-1)$ respectively, and let $b_{p}=1-\gamma(0) \cos (\delta(p) \log q)$ be the bottom of (the real part of) the $L^{p}$ spectrum of $\mathcal{L}$. 
Either by using the pointwise estimate of Lemmas 3 and 4, or by means of Fourier analysis techniques as done in CMS, it may be shown that

$$
\left\|h_{t}\right\|_{p} \sim \begin{cases}\min \left\{1, t^{1 /\left(2 p^{\prime}\right)}\right\} \exp \left(-b_{p} t\right) & \text { if } 1 \leq p<2, \\ \min \left\{1, t^{-3 / 4}\right\} \exp \left(-b_{2} t\right) & \text { if } p=2, \\ \min \left\{1, t^{-3 / 2}\right\} \exp \left(-b_{2} t\right) & \text { if } 2<p \leq+\infty\end{cases}
$$

and it follows that

$$
\left\|h_{t}\right\|_{p}^{-p} \sum_{|x|=d-1} h_{t}^{p}(x) \sim \frac{d^{p}}{t^{p}\left[d^{2}+\gamma^{2}(0) t^{2}\right]^{p / 4}} \exp \left\{p g_{p}(t, d)\right\}
$$

where

$g_{p}(s, t)=-\gamma(i \delta(p)) t+\delta(p) \log q s+\left(s^{2}+\gamma^{2}(0) t^{2}\right)^{1 / 2}+s \log \left(\frac{\gamma(0) t}{s+\left[s^{2}+\gamma^{2}(0) t^{2}\right]^{1 / 2}}\right)$.

It is easily seen that

- if $1 \leq p<2$, then the maximum value of $g_{p}(s, t)$ is 0 and is attained at the point $\bar{s}=R_{p} t$ where $R_{p}=\left(q^{p}-q^{p^{\prime}}\right) / q+1$;

- if $p \geq 2$, then $g_{p}(s, t)$ is decreasing for $s \geq 0$.

Taking this into account, computations similar to those used in the proof of Theorem 1 allow us to deduce the following.

(i) Let $1 \leq p<2$. If $r(t)$ is any function such that $r(t) / t^{1 / 2} \rightarrow+\infty$ as $t \rightarrow+\infty$, then

$$
\left\|h_{t}\right\|_{p}^{-p} \sum_{R_{p} t-r(t) \leq|x| \leq R_{p} t+r(t)} h_{t}(x)^{p} \rightarrow 1 \quad \text { as } t \rightarrow+\infty .
$$

(ii) Let $p=2$. If $r_{1}(t) / t^{1 / 2} \rightarrow 0$ and $r_{2}(t) / t^{1 / 2} \rightarrow+\infty$ as $t \rightarrow+\infty$, then

$$
\left\|h_{t}\right\|_{2}^{-2} \sum_{r_{1}(t) \leq|x| \leq r_{2}(t)} h_{t}(x)^{2} \rightarrow 1 \quad \text { as } t \rightarrow+\infty
$$

(iii) Let $2<p<+\infty$. If $r_{3}(t) \rightarrow+\infty$ as $t \rightarrow+\infty$, then

$$
\left\|h_{t}\right\|_{p}^{-p} \sum_{|x| \leq r_{3}(t)} h_{t}(x)^{p} \rightarrow 1 \quad \text { as } t \rightarrow+\infty
$$

(iv) If $p=+\infty$, the maximum of $h_{t}(x)$ is attained at $o$ and

$$
\left\|h_{t}\right\|_{+\infty}^{-1} \sup _{|x| \geq r_{3}(t)} h_{t}(x) \rightarrow 0 \quad \text { as } t \rightarrow+\infty .
$$

We note that the $L^{p}$ heat propagation on symmetric spaces is studied in [AJ]. The behaviour described by (i)-(iv) above corresponds exactly to what is obtained therein.

Proof of Theorem 2. The proof follows the lines of that of Theorem 1 above. Since $h_{t}^{\mathbb{Z}}$ is even and has total sum $1,(2)$ is equivalent to the validity of

$$
\sum_{0 \leq d \leq r_{1}(t)} h_{t}^{\mathbb{Z}}(d) \rightarrow 0 \quad \text { as } t \rightarrow \infty
$$


and

$$
\sum_{d \geq r_{2}(t)} h_{t}^{\mathbb{Z}}(d) \rightarrow 0 \quad \text { as } t \rightarrow \infty
$$

By Lemma 3, we have the estimate

$$
h_{t}^{\mathbb{Z}}(d) \leq C \frac{1}{\left(d^{2}+t^{2}\right)^{1 / 4}} e^{g_{Z}(d, t)}, \quad \forall d \in \mathbb{Z}^{+} \text {and } t \geq 1,
$$

where $g_{\mathbb{Z}}(s, t)$ is defined by

$$
g_{\mathbb{Z}}(s, t)=-t+\left(s^{2}+t^{2}\right)^{1 / 2}+s \log \left(\frac{t}{s+\left[s^{2}+t^{2}\right]^{1 / 2}}\right) .
$$

Differentiating twice with respect to $s$ yields

$$
g_{\mathbb{Z}}^{\prime \prime}(s, t)=-\frac{1}{\left(s^{2}+t^{2}\right)^{1 / 4}} \leq-\frac{1}{\sqrt{2} t}
$$

for all $s \leq t$. Therefore, in the same range of $s$

$$
g_{\mathbb{Z}}^{\prime}(s, t)=\int_{0}^{s} g_{\mathbb{Z}}^{\prime \prime}(u, t) d u \leq-\frac{s}{\sqrt{2} t} .
$$

Since $g_{\mathbb{Z}}(0, t)=0$, by the mean value theorem,

$$
g_{\mathbb{Z}}(s, t)=\int_{0}^{s} g_{\mathbb{Z}}^{\prime}(u, t) d u \leq-\frac{s^{2}}{2 \sqrt{2} t}, \quad s \leq t .
$$

On the other hand, if $s \geq t$, we expand around $s=t$ and write

$$
\begin{aligned}
g_{\mathbb{Z}}(s, t)=g_{\mathbb{Z}}(t, t)+g_{\mathbb{Z}}^{\prime}(t, t)(s-t) & +\int_{t}^{s} g_{\mathbb{Z}}^{\prime \prime}(u, t)(s-u) d u \\
& \leq-\frac{t}{2 \sqrt{2}}-\frac{1}{\sqrt{2}}(s-t) .
\end{aligned}
$$

Then, proceeding as in the proof of Theorem 1, we have

$$
\begin{aligned}
\sum_{0 \leq d \leq r_{1}(t)} h_{t}^{\mathbb{Z}}(d) & \leq C \int_{0}^{r_{1}(t)} \frac{1}{\left(d^{2}+t^{2}\right)^{1 / 4}} e^{g_{\mathbb{Z}}(d, t)} \\
& \leq \frac{C}{\sqrt{t}} \int_{0}^{r_{1}(t)} e^{-s^{2} / 2 \sqrt{2} t} d s=C \int_{0}^{r_{1}(t) / \sqrt{t}} e^{-u^{2} / 2 \sqrt{2}} d u
\end{aligned}
$$

and since the right-hand side tends to 0 as $t \rightarrow+\infty$, (8) follows. Similarly,

$$
\begin{aligned}
\sum_{d \geq r_{2}(t)} h_{t}^{\mathbb{Z}}(d) & \leq C \int_{r_{2}(t)}^{+\infty} \frac{1}{\left(s^{2}+t^{2}\right)^{1 / 4}} e^{g_{\mathbb{Z}}(s, t)} d s \\
& =C\left(I_{1}(t)+I_{2}(t)\right),
\end{aligned}
$$

where $I_{1}$ is the integral over the interval $\left(r_{2}(t), t\right)$ and $I_{2}$ that over $(t,+\infty)$. Using (9) we may estimate

$$
\begin{aligned}
I_{1}(t) & \leq \frac{C}{\sqrt{t}} \int_{r_{2}(t)}^{t} e^{-s^{2} / 2 \sqrt{2} t} d s \\
& =C \int_{r_{2}(t) / \sqrt{t}}^{\sqrt{t}} e^{-u^{2} / 2 \sqrt{2}} d u \rightarrow 0 \quad \text { as } t \rightarrow+\infty .
\end{aligned}
$$


By (10) we also have

$$
\begin{aligned}
I_{2}(t) & \leq C e^{-t / 2 \sqrt{2}} \int_{t}^{+\infty} \frac{1}{\sqrt{s}} e^{-(s-t) / \sqrt{2}} d s \\
& \leq C \frac{e^{-t / 2 \sqrt{2}}}{\sqrt{t}} \int_{\sqrt{t}}^{+\infty} e^{-u / \sqrt{2}} d u
\end{aligned}
$$

and the right-hand side is exponentially decreasing as $t$ tends to infinity. This shows that $\left(8^{\prime}\right)$ holds, as required to complete the proof of the theorem.

\section{ACKNOWLEDGMENTS}

This paper is the outgrowth of joint work of the second named author with Michael G. Cowling and Stefano Meda, and we are grateful to them for many helpful discussions. We also wish to thank Jean-Philippe Anker for suggesting that we look at the $L^{p}$ heat propagation.

\section{REFERENCES}

[AJ] J.-Ph. Anker and L. Ji, Heat kernel and Green function estimates on noncompact symmetric spaces, preprint.

[AS] J.-Ph. Anker and A.G. Setti, Asymptotic finite propagation speed for heat diffusion on certain Riemannian manifolds, Jour. Funct. Anal. 103 (1992), 50-61. MR 93c:58208

[B] M. Babillot, A probabilistic approach to heat diffusion on symmetric spaces, Jour. Theor. Probability 7 (1994), 599-607. MR 95h:58143

[CMS] M. Cowling, S. Meda and A.G. Setti, Estimates for functions of the Laplace operator on homogeneous trees, Trans. Amer. Math. Soc., to appear. CMP 99:04

[D] E.B. Davies, Heat Kernels and Spectral Theory, Cambridge University Press, 1989. MR 90e:35123

[E] A. Erdélyi, Asymptotic Expansions, Dover, 1956. MR 17:1202c

[MS] G. Medolla and A.G. Setti, The wave equation on homogeneous trees, Ann. Mat. Pura e Appl. CLXXVI (1999), 1-27.

[P] M.M.H. Pang, Heat kernels on graphs, J. London Math. Soc. 47 (1993), 50-64. MR 93k:60177

Dipartimento di Matematica, Politecnico di Milano, via Bonardi 9, I-20133 Milano, ITALY

Current address: I.T.I.S Hensemberger, via Berchet, I-20052 Monza, MI, Italy

E-mail address: guimed@iol.it

Dipartimento di Scienze Chimiche Fisiche e Matematiche, Università dell'Insubria Polo di Como, via Lucini 3, I-22100 Como, Italy

E-mail address: setti@fis.unico.it 